DOI: $\square$ https://doi.org/10.15407/techned2020.06.015

\title{
MAGNETIC FIELD SHIELDING OF UNDERGROUND POWER CABLE LINE BY H-SHAPED SHIELD
}

Journal

Publisher

ISSN

Issue

Pages
Tekhnichna elektrodynamika

Institute of Electrodynamics National Academy of Science of Ukraine 1607-7970 (print), 2218-1903 (online)

No 6, 2020 (November/December)

$15-20$

\section{Author}

\section{I.M. Kucheriava}

Institute of Electrodynamics of National Academy of Sciences of Ukraine, pr. Peremohy, 56, Kyiv, 03057, Ukraine, e-mail: rB.irinan@gmail.com

\section{Abstract}

In the article the magnetic field distributions, generated by underground extra-high voltage (330 $k V$ ) three-phase power cable line in the environment, in particular near the cables in the trench and on the ground, are analyzed for using of $\mathrm{H}$-shaped shield made of different materials including aluminum, low carbon steel and non-oriented grain steel. As shown, the best shielding effectiveness is realized by aluminium shield. The $\mathrm{H}$-shaped shield made of high-conducting non-magnetic materials is proposed to use in order to mitigate the magnetic field level on the ground down to regulated nonhazardous values. References 14, figures 7 .

Key words: underground extra-high voltage power cable line, trench cable laying, $\mathrm{H}$-shaped shield, ferromagnetic shield, high-conducting materials, ecological safety, computer modeling. 


\section{References}

1. Electrical power cable engineering. Third Edition, ed. by W.A. Thue. CRC Press, 2011. 460

p.

2. Doronin M.V., Greshniakov G.V., Korovkin N.V. Magnetic shields of special design. Nauchno -tekhnicheskie vedomosti Sankt-Peterburgskogo politekhnicheskogo universiteta 2017. Vol. 23. No 1. Pp. 124-133. DOI:

https://doi.org/10.18721/ JEST.230112

(Rus)

3. Kucheriava I.M. Shielding of underground extra-high voltage cable line by plane ferromagnetic shield. Tekhnichna Elektrodynamika. 2019. No 6. Pp. 13-17. (Rus) DOI: https:// doi.org/10.15407/techned2019.06.013

4. Shcherba A.A., Podoltsev O.D., Kucheriava I.M. The magnetic field of underground $330 \mathrm{kV}$ cable line and ways for its reduction. Tekhnichna Elektrodynamika. 2019. № 5. Pp. 3-9. (Rus) DOI:

https://doi.org/10.1540

7/techned2019.05.003

5. De Wulf M., Wouters P., Sergeant P., Dupré L., Hoferlin E., Jacobs S., Harlet P. Electromagnetic shielding of high-voltage cables. Journal of Magnetism and Magnetic Materials .2007. No 316. Pp. 908-911. DOI:

https://doi.org/10.1016/j.jmmm.2007.03.137

6. Gille A., Beghin V., Geerts G., Hoeffelman J., Liémans D., Van Gucht K. Double 150 kV link, $32 \mathrm{~km}$ long, in Belgium: design and construction. Cigre Session. 2004. Paper B1-305. 8 p.

7. Del Pino J.C., Cruz-Romero P., Serrano-Iribarnegaray L. Impact of electromagnetic losses in closed two-component magnetic shields on the ampacity of underground power cables. Progres $s$ in Electromagnetics Research

. 2013. Vol. 135. Pp. 601-625. DOI:

https://doi.org/10.2528/PIER12112303

8. Electric installation code. Kyiv: Minpalivo Ukrainy, 2010. 776 p. (Ukr)

9. Conti R., Donazzi F., Maioli P., Rendina R., Sena E.A. Some Italian experiences in the utilization of HV underground cable systems to solve local problems due to magnetic field and other environmental issues. Cigre Session. 2006. Paper C4-303.

10. Comsol multiphysics modeling and simulation software. URL: http://www.comsol.com/ (accessed at 25.03.2020)

11. Lyach V.V., Molchanov V.M., Santatskii V.G., Kvitsinskii A.A. 330 kV cable line: some aspects of designing. Promelektro. 2009. No 6. Pp. 27-33. (Rus)

12. Bravo-Rodriguez J.C., Del-Pino-Lopez J.C., Cruz-Romero P. A survey on optimization techniques applied to magnetic field mitigation in power systems: review. Energies. 2019. No 12. 1332.20 p. DOI: https://doi.org/10.3390/en12071332

13. Podoltsev A.D., Kucheriava I.M. Multiphysics modeling in electrical engineering. Kyiv: Institute of Electrodynamics, Ukrainian Academy of Sciences, 2015. 305 p. (Rus) 
14. Del-Pino-Lopez J.C., Cruz-Romero P., Dular P. Parametric analysis of magnetic field mitigation shielding for underground power cables. The Renewable Energy \& Power Quality Journal

(RE\&PQJ). March 2007. Vol. 1. No

5. Pp. 519-526. DOI:

https://doi.org/

10.24084/repqj05.326

PDF

@ $\Theta \Theta \odot$

This work is licensed under a Creative Commons Attribution-NonCommercial-NoDerivatives 4.0 International License 